\title{
Science, Politics and Hydroxychloroquine
}

\author{
John Somberg
}

Coronavirus disease 2019 (COVID-19) has remained a persistent public health problem with significant worldwide impact. In the USA there have been 3.9 million confirmed cases with 143,000 confirmed deaths due to COVID-19. The journal Science reports that there have been 22,000 scientific papers written on COVID-19 and we currently have 2,000 clinical trials underway. One would think with the number of cases and the number of clinical investigations underway, the issue of the effectiveness of a very old drug such as hydroxychloroquine would be resolved. However, to date the use of hydroxychloroquine in COVID-19 treatment remains highly controversial. Its use has markedly declined and some states are using medical boards to inhibit physicians from prescribing.

Hydroxychloroquine is a very old drug, first approved for clinical use in the USA in 1955. It has less side effects than chloroquine, and is the preferred treatment. It is on the World Health Organizations list of essential medications. It is the 128th most commonly prescribed medication in the USA. For its principle use, malaria prophylaxis, there is far greater prescription worldwide. It is a well-tolerated medication for the treatment of rheumatoid arthritis and lupus. Its principle risk, though infrequent, is the precipitation of life-threatening arrhythmias that result from significant QTc prolongation that occurs in some patients. The drug is a weak inhibitor of the potassium Ikr channel, and this can cause life-threatening arrhythmias. Women with significant QTc prolongation at baseline and patients with occult channelopathies can be at risk. Still in the overwhelming majority of patients the drug is well tolerated, e.g., has been on the US market for 65 years.

On the basis of case reports and uncontrolled studies, early information suggested a benefit of hydroxychloroquine uses in COVID-19 patients. In vitro studies found suppression of virus replication with hydroxychloroquine. This led to the Food and Drug Administration (FDA) issuing an emergency use authorization on March 28 of this year. In May the Lancet published a large international study that found hospitalized patient with COVID-19 were at higher risk of death by $30 \%$. However, the Lancet retracted the study based on serious methodological flaws as pointed out by over 120 clinical researchers. In fact, after the criticism one author (the source of the database) refused to provide the data for a second peer review. Another study by Oxford University

Manuscript submitted July 22, 2020, accepted July 24, 2020

Published online August 1, 2020

Cardiology and Pharmacology, Rush University, Chicago, IL 60612, USA. Email: John_Somberg@rush.edu

doi: https://doi.org/10.14740/cr1140 researchers found at a mid-study evaluation, a lack of benefit of the hydroxychloroquine when treatment started 9 days on average after onset of symptoms. Additionally, a study reported in the British Medical Journal found no benefit on mortality, but found some symptoms to attenuate. Following these reports, FDA revoked the emergency authorization. This decision paralleled the World Health Organization discontinuation of the drug in the Solidarity trial. The Recovery Trial in the UK showed no benefit of hydroxychloroquine in hospitalized patients with severe COVID-19. The question arose as to the timing of treatment and COVID-19 severity as factors affecting success of therapy.

However, subsequently there have been significant studies that have found contradictory evidence. A study from Mount Saini Hospital in New York found that after adjusting for confounding variables, patients had a $47 \%$ lower mortality on hydroxychloroquine. This has been reported similarly in a nonrandomized 2,600 patient study from Henry Ford Hospital in Detroit. There was a $50 \%$ reduction in mortality in patients on hydroxychloroquine than those on no therapy. While nonrandomized, this large study suggests a benefit of treatment and certainly no harm from hydroxychloroquine.

So what should clinical scientists and physicians make of the current state of knowledge? It is incomplete, but what information we know from reliable sources supports the benefits of hydroxychloroquine. Its benefits seem to be most notable when started early, in less severely sick patients who are not yet hospitalized, although their appears benefit from therapy as the disease progresses. Certainly the FDA should once again, encourage clinical studies and the clinical use of hydroxychloroquine for early onset of COVID-19 should be available, as well as for prophylaxis on very high-risk individuals such as COVID-19 testing site workers and hospital personnel exposed to contagious patients. With the vast number of cases, it would seem that well organized studies by National Institutes of Health (NIH), HSS, state governments and academic institutions should be able to resolve the safety and effectiveness of hydroxychloroquine.

For those physicians whose patients want the therapy, caution in patient selection along the lines employed for all QTc prolonging agents needs to be employed. Baseline QTc must be obtained. Patients with excessive QT prolongation (460 to $500 \mathrm{~ms}$ and greater) should not receive the therapy. Physiologic conditions that promote arrhythmogenicity of QT prolongation such as bradycardia, hypokalemia, hypomagnesemia and congenial QT prolongation syndromes must be avoided, and patients with these conditions should be excluded.

Clinical science needs to prevail. Political bias has no place in science and the evaluation of hydroxychloroquine as a COVID-19 therapeutic option. 


\section{Acknowledgments}

None to declare.

\section{Financial Disclosure}

None to declare.

\section{Conflict of Interest}

None to declare.

\section{Data Availability}

The author declares that data supporting the findings of this study are available within the article. 